\title{
Recent Progress in Benzocyclobutene Related Polymers
}

\author{
Junxiao Yang, Yawen Huang and Ke Cao
}

Additional information is available at the end of the chapter

http://dx.doi.org/10.5772/48129

\section{Introduction}

The developing history of polymer materials tells that the invention of novel polymers with excellent performance is generally originated from the synthesis of new polymerizable monomers. Among kinds of monomers, benzocyclobutene (BCB) has attracted much interest because of its unique molecular structure and polymer properties (1-2). The structure of $B C B$ is shown as following with the numbering system, the CA name of which is bicyclo[4,2,0]octa-1,3,5-triene. In terms of the four-membered ring structure, BCBs are able to transform into the reactive $o$-quinodimethane, also known as $o$-xylylene, upon heating at around $200{ }^{\circ} \mathrm{C}$. This very reactive specie readily undergoes inter- and intramolecular DielsAlder reaction with various dienophiles to produce polycyclic compounds with high stability, such as cyclooctadiene. On the other hand, the polymerization of $o$ quinodimethane similar to that of 1,3-diene would give linear polymers. Additionally, the reaction of $\mathrm{BCB}$ does not require any catalyst and release any small molecules, thus hardly introducing any impurities into materials. These features undoubtedly offer BCB based materials several superiorities in several specific applications, such as microelectronic applications, optic applications, electrical applications, etc.

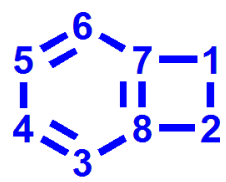

Nowadays, hundreds of BCB based structures have been synthesized, which constituted abundant resource for producing high performance and functional materials. Varying the structures and properties of BCB building blocks and constructing methods would afford materials with tunable or attractive properties and performance. Early prepared BCB 
monomers were mainly BCB-bicapped structures. As described previously, these compounds can be utilized to generate reactive oligomers which are processable intermediates to prepare polymeric materials. In its most basic statement, the concept of reactive oligomers involves insitu conversion of low molecular weight compounds into high molecular weight materials. Reactive oligomers are in fact a very old technology with the earliest examples being found in the preparation of organic coatings. This technology however is limited to inferior filmforming properties. To address this problem, more recently, new structures were introduced to make a modification of present structures. Of them, poly (vinyl-benzocyclobutene) has attracted increased attention (3). In different with the polymers prepared from BCB-dicapped monomers, the BCB unit were placed on the side chains in poly (vinyl-benzocyclobutene). Thanks to their well-controllable structures, properties and performance, these polymers show potential application in nanoparticles preparation, surface modification, apart from microelectronic. On the other hand, early polybenzocylobutenes also suffer from relatively low thermal stability. As a respond to the requirement of high thermal resistance, a new class of polybenzocylobutenes, benzocyclobutene-siloxane resins, has been extensively studied. Through introducing of thermostable polysiloxane chains, remarkable enhancement in thermal resistance has attained.

Overall, since Finkelstein reported the synthesis of a BCB derivative in 1909 for the first time, the research on BCB has experienced rapid development. The research field has extended from chemistry to materials even physics. Correspondingly, the research targets have also shown a transition from low molecular weight molecules to high molecular weight materials. Furthermore, the applications of BCB-based materials have extended from dielectrics to materials modification, nanostructure construction, and so on. All of these trends suggest a prospective future of BCB related materials. Previously, several excellent reviews have dealt with the development of $B C B$ related science (1-2). More recently, although new reviews involving the $\mathrm{BCB}$ chemistry have been reported (4-5), the progress in $\mathrm{BCB}$ related polymers has not been reviewed until now. In this chapter, we will present a review of BCB from 1996, which will concern about two types of newly developed BCBbased materials, poly (vinyl-BCB) and BCB-siloxane polymers.

\section{Vinyl-benzocyclobutene}

From a closer look of the structures of the classical BCB resins prepared from BCB di-capped monomers, it can be noted that these cross-linked polymers were prepared by directly taking use of intramolecular Diels-Alder reaction and the functionality of these monomers was more than 2. As a result, cross-linking and polymerization take place simultaneously. Because they lack well-defined structures, conventional BCB-based polymers are difficult to provide controllable properties. Consequently, their applications in many areas are limited. In addition, the network structures are mainly constructed by the polycyclic structure. This highly rigid structure is in general unfavorable for generating higher cross-linking degree.

Polyolefins represent an important class of polymer whose structure could be conveniently controlled by sophisticated methods. More importantly, reactive functional groups are able 
to be introduced into polyolefins conveniently (6-7). Such built-in cross-linkable functionality leads to two-step crosslinking, offering substantial advantages over alternative two-component mixtures or one-step formation of cross-linked, network solids. First of all, chain growth and cross-linking reactions become independent of one another, either expanding the materials application range or improving the processability of thermally stable polymers. Second, this cross-linking protocol provides complete control over the chemical structure of the primary polymer, network structures and physical/chemical properties of the matrix. Meanwhile, the cross-linking agent is incorporated as a comonomer into the backbone of the relative high molecular weight polymers, allowing the cross-linking density to be directly related to the monomer composition. Hence, constructing new BCB polymers with polyolefin backbone are expected to open a new road towards controllable structure of BCB-based materials.

\subsection{Preparation and properties of vinyl-BCB polymers}

\subsubsection{Poly (4-vinyl benzocyclobutene)}

As a typical vinyl-BCB, 4-vinyl benzocyclobutene (4-VBCB) as shown in Scheme 1, was firstly synthesized by Endo et al. (8) and more recently by Hawker et al. (9). Of several wellestablished polymerization protocols, free radical and anionic methods have been so far demonstrated available for the polymerization of 4 -VBCB. Copolymerization of vinyl-BCB with other vinyl monomers such as styrene, methyl methacrylate, and $n$-butyl acrylate, were proved to be a controllable procedure, leading to random incorporation of the reactive $\mathrm{BCB}$ units. For example, So et al. reported the copolymerization of 4 -VBCB with styrene initiated by benzoyl peroxide (10). As-prepared polystyrene-co-4-VBCB random copolymer contains $26 \mathrm{~mol} \%$ of $4-\mathrm{VBCB}$, with $M_{n}$ of $23104 \mathrm{~g} / \mathrm{mol}$ and PDI of 1.83 . Harth et al $(9,11)$ employed nitroxide mediated living radical polymerization to prepare $4-\mathrm{VBCB} /$ styrene random copolymers (Scheme 1). It was pointed out that at molecular weight less than 120,000 the PDIs for these random copolymers were 1.08-1.16, which slightly increased to 1.19-1.26 for molecular weights above 200,000. Using living anionic polymerization initiated by secbutyllithium and $n$-butyllithium, Baskaran et al. have prepared poly (4-VBCB) successfully (3). The polymerization was allowed to proceed at $25^{\circ} \mathrm{C}$ for $15 \mathrm{~h}$. A linear first-order semilogarithmic time-conversion plot was obtained, which indicated the absence of termination reactions during the polymerization of 4 -VBCB at room temperature. The molecular weight of resulted poly $(4-\mathrm{VBCB})$ was tailored by varying the amount of initiators and the maximum $M_{n}$ can reach as high as 58,000 in their experiments. In addition, all of the prepared poly (4-VBCB)s exhibited narrow molecular weight distributions $(<1.1)$.

In the above examples, 4-VBCB was generally used as a reactive functional group, which acts as active sites for cross-linking reactions. Taking poly(styrene-r-4-VBCB) as an example, the crosslinking structure is composed of cyclic and linear structure (Scheme 2) as suggested in many reports $(10,12,13)$. Moreover, the crosslinking of these polymers were fulfilled by the Diels-Alder reaction of $\mathrm{BCB}$ with high atom economy. 

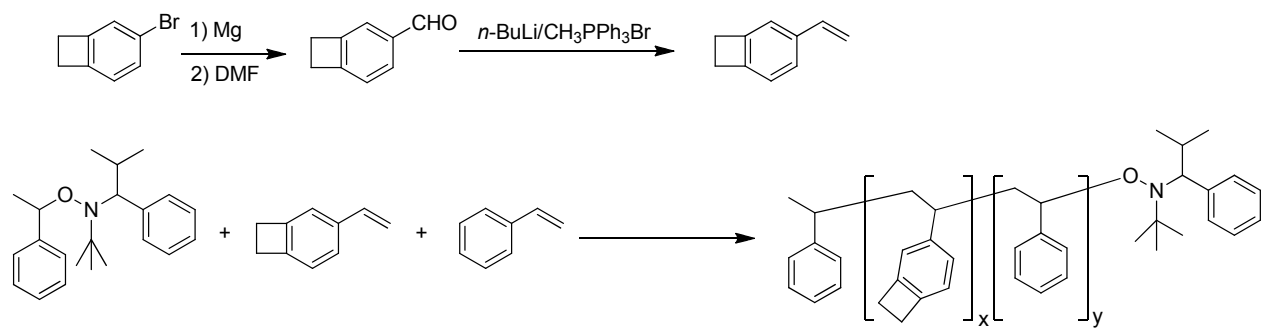

Scheme 1. Synthesis route to $1-\mathrm{VBCB}$ and its nitroxide-mediate polymerization.

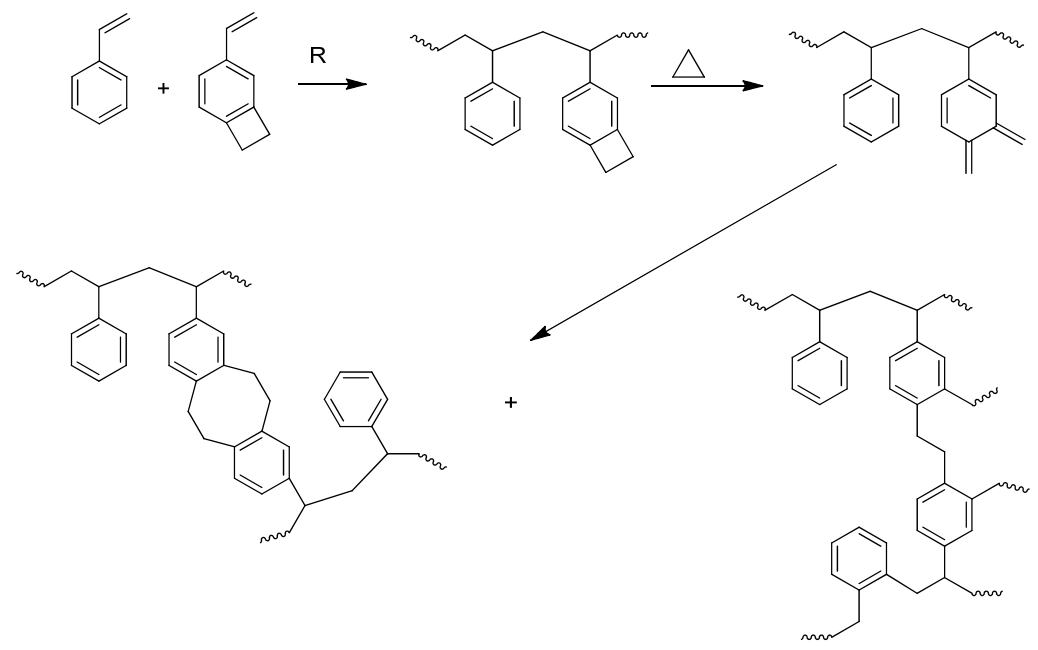

Scheme 2. Copolymerization of styrene and 4-VBCB and the cross-linking structure of poly (St-r-4$\mathrm{VBCB})$

The cross-linking of polyolefins by means of BCB presents a considerable significance in stabilizing the polymeric matrix, including enhancing the glass transition temperature $\left(T_{g}\right)$, thermal stability and mechanical stability. Resulting cross-linked materials can be potentially exploited in the design of high voltage cable insulation, biomedical joint replacement parts, hot-water piping, and etc (7). Recently, it has been reported for other applications including generation of cross-linked/monomolecular polystyrene nanoparticles $(9,14,15)$, modification of solid surfaces (16-17), stabilization of nanostructures, thermally stable diblock copolymers in data storage and nanoscale templating (11).

On the basis of 4 -VBCB and derivative poly (4-VBCB), Yang et al. have designed and synthesized a series of new vinyl-BCBs (Scheme 3), including 4-vinylsilylbenzocyclobutene (4-DMVSBCB) (18), 1-vinylsilylbenzocyclobutene (1-DMVSBCB), benzocyclobutene-4-yl acrylate (4-ABCB) (19-20), and benzocyclobutene-1-yl acrylate (1-ABCB). 4-DMVSBCB was synthesized conveniently by Grignard reaction of 4-bromobenzocyclobutene with vinyldimethylchlorosilane. With the same procedure, 1-DMVSBCB could be synthesized in good yield. 4-ABCB was synthesized through a facile esterification reaction. The detailed 
routes are outlined in Scheme 3. These new monomers, especially 4-DMVSBCB showed different polymerization behavior compared with $4-\mathrm{VBCB}$, probably caused by the incorporation of silicon containing group.

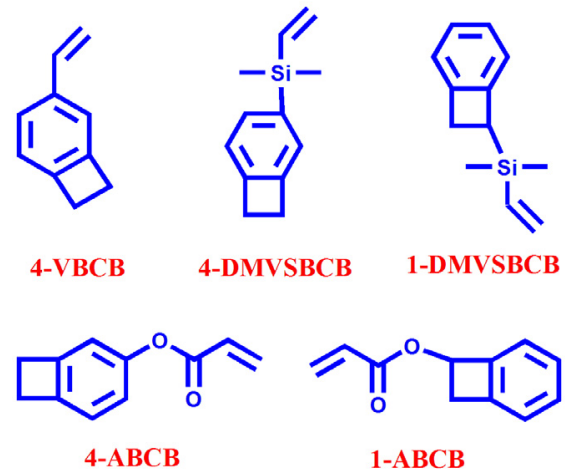

Scheme 3. Chemical structure of several new vinyl-BCBs

\subsubsection{Poly (4-vinylsilylbenzocyclobutene)}

As a vinylsilane, the polymerization of 4-DMVSBCB with different groups has been studied extensively. In fact, it exhibited almost the same behavior with another vinylsilane compound, 4-dimethylvinylphenylsilane (4-DMVSPh), which was reported to polymerize by the radical, anionic and coordination methods. The homo-polymerization of 4-DMVSBCB is sluggish when initiated by BPO or AIBN, only giving trace amount of poly(4-DMVSBCB) with $M_{n}$ between 7500 to $8900 \mathrm{~g} / \mathrm{mol}$. Theoretically, it is probably attributed to the lack of conjugated structure and the electron-donating effect of silicon group. Contrastively, the copolymerization of 4-DMVSBCB with conventional $\alpha$-olefins was successfully performed with low to moderate yield ( $<50 \%)$. However, the incorporation ratio of 4 -DMVSBCB is still low ( $<10 \mathrm{~mol} \%)$ and hard to increase even employing high concentration of 4-DMVSBCB (Table 1). Thus, alternative polymerization method to enhance polymerization activity and incorporation ratio in conventional polyolefins is required.

As compared with radical polymerization, the anionic polymerization of 4-DMVSBCB affords well-controlled and high molecular weight, and relatively low polydispersity. Anionic homo-polymerization of 4-DMVSBCB was performed using $n$-BuLi and sec-BuLi as initiators (Scheme 4). White solid polymers with molecular weight $\left(M_{n}\right)$ of $9800-26,000$ $\mathrm{g} / \mathrm{mol}$ and PDI in the range of 1.6-1.8 were obtained in excellent yields (up to $94.5 \%$ ). Structural studies have shown that $\mathrm{d} \pi-\mathrm{p} \pi$ interactions in the vinyl-silicon bond give resonance contributions to the vinyl-silicon bond, which provides the polarization of the vinyl group necessary for anionic polymerization. Brooks et al. has reported that RLi adds to vinylsilanes so as to generate a secondary lithium compound. Furthermore, as noted before, the incorporation of aryl groups, especially phenyl, is more favorable to enhance anionic polymerization activity than that of alkyl groups. Related studies revealed that the aryl 
groups on silicon atom could provide silicon atom with enhanced positive charge and $\alpha$ stabilizing effect due to the electron-withdrawing property of aryl groups.

\begin{tabular}{cccccccc}
\hline Entry & $\begin{array}{c}\text { Initiator } \\
\left(10^{-2} \mathrm{mmol}\right)\end{array}$ & $\begin{array}{c}4 \text {-DMVSBCB } \\
(\mathrm{mmol})\end{array}$ & $\begin{array}{c}\text { comonomer } \\
(\mathrm{mmol})\end{array}$ & $\begin{array}{c}\text { temp } \\
\left({ }^{\circ} \mathrm{C}\right)\end{array}$ & $\begin{array}{c}\text { time } \\
(\mathrm{h})\end{array}$ & $\begin{array}{c}\text { content of 4- } \\
\text { DMVSBCB } \\
(\mathrm{mol} \%)\end{array}$ & $\begin{array}{c}\text { yield } \\
(\%)\end{array}$ \\
\hline $\mathbf{1}$ & AIBN 8.64 & 4.28 & & $60-65$ & 60 & & \\
$\mathbf{2}$ & BPO 5.08 & 2.64 & & $75-80$ & 60 & & \\
$\mathbf{3}$ & AIBN 6.35 & 2.65 & $\mathrm{St}, 2.69$ & 60 & 48 & 5.3 & $45 \%$ \\
$\mathbf{4}$ & AIBN 8.34 & 3.19 & $\mathrm{St}, 1.61$ & 60 & 48 & 7.3 & $36 \%$ \\
$\mathbf{5}$ & AIBN 8.74 & 4.26 & St, 1.06 & 60 & 48 & 9.5 & $29 \%$ \\
$\mathbf{6}$ & AIBN 7.92 & 2.68 & 4-BrSt, 2.58 & 60 & 48 & 13 & $48 \%$ \\
$\mathbf{7}$ & BPO 7.38 & 3.19 & St, 1.73 & 75 & 36 & & $38 \%$ \\
$\mathbf{8}$ & BPO 8.68 & 2.16 & MMA, 2.21 & 75 & 36 & & \\
$\mathbf{9}$ & BPO 11.46 & 3.22 & VA, 3.16 & 75 & 36 & & \\
\hline
\end{tabular}

Table 1. Polymerization and Copolymerization of 4-DMVSBCB

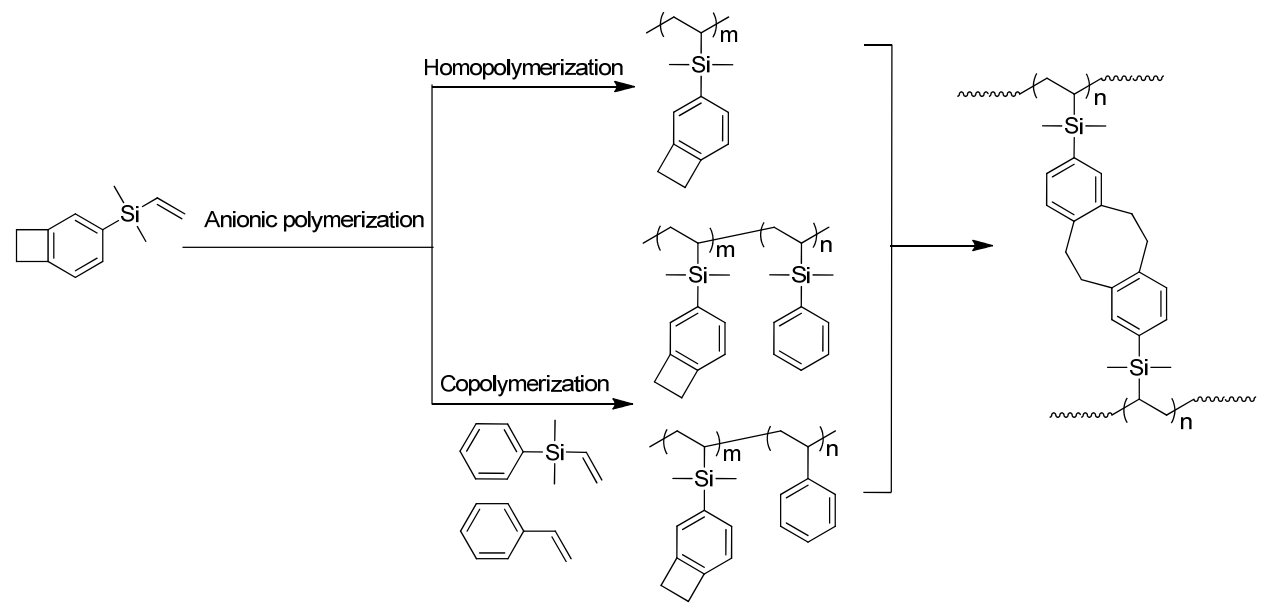

Scheme 4. Anionic homo and copolymerization of 4-DVMSBCB

Poly (4-DMVSBCB) shows several outstanding properties. First, it shows superior film forming property. A problem encountered in the film forming process for the low molecular weight polymer is its low viscosity. One solution to this issue was to pre-crosslink the polymer in prior to film forming. In the film forming process of the polymer derived from $\mathrm{BCB}$ bis-capped monomer, the pre-crosslinking process is hard to control as gel formation occurred immediately when gel point reached. In comparison, pre-crosslinking process of poly (4-DMVSBCB) is controllable because its polymerization and cross-linking processes were separated and the incorporating ratio of BCB cross-linkable functionality into polyolefins was controllable. Second, TGA in air shows that the initial decomposition temperature $\left(T_{0}\right)$ and $5 \mathrm{wt} \%$ weight loss temperature $\left(T_{5} \%\right)$ of poly (4-DMVSBCB) are about 
$318^{\circ} \mathrm{C}$ and $400{ }^{\circ} \mathrm{C}$, respectively. In particular, the equilibrium rate of mass loss for the crosslinked poly (4-DMVSBCB) was $0.95 \mathrm{wt} \% / \mathrm{h}$, which is lower than that of Cyclotene ${ }^{\mathrm{TM}}$ resins. The dielectric constant of subtle cross-linked poly (4-DMVSBCB) is in the range of 2.41 2.45 from $5 \mathrm{MHz}$ to $20 \mathrm{MHz}$. This value is lower than that of most polymeric matrix. Third, a film fabricated from highly cross-linked poly (4-DMVSBCB) shows a greatly small roughness. These properties show that it could be applied in some interconnect fabrication processes which are implemented at temperature as high as $350{ }^{\circ} \mathrm{C}$.

The copolymerization of 4-DMVSBCB with styrene and DMVSPh was also performed (Scheme 4). It is worth noting that the ratios of 4-DMVSBCB to 4-DMVSPh in copolymers showed well accordance with the feed ratios due to their similar structure and reactivity (Table 2). In contrast, when copolymerizing 4-DMVSBCB with styrene, the latter show higher reactivity and incorporation ratio than the former because of the conjugation effect of phenyl group. More importantly, the incorporation of styrene or DMVSPh into poly (4DMVSBCB) did not lead to obvious reduction in thermal resistance, even when 1:1 of comonomers to 4-DMVSBCB was used.

\begin{tabular}{cccccc}
\hline Entry & initiator I & {$[\mathrm{I}] /[\mathrm{M}]^{a}$} & $M_{\mathrm{n}}{ }^{b}$ & $M_{\mathrm{w}} / M_{\mathrm{n}}$ & yield $^{c}(\%)$ \\
\hline $\mathbf{1}$ & $n-\mathrm{BuLi}$ & $1.9 \times 10^{-3}$ & 13900 & 1.62 & 92 \\
$\mathbf{2}$ & $n-\mathrm{BuLi}$ & $1.2 \times 10^{-3}$ & 14500 & 1.71 & 89 \\
$\mathbf{3}$ & $n-\mathrm{BuLi}$ & $9.4 \times 10^{-4}$ & 22400 & 1.77 & 93 \\
$\mathbf{4}$ & $n-\mathrm{BuLi}$ & $5.7 \times 10^{-4}$ & - & - & 27 \\
$\mathbf{5}$ & $n-\mathrm{BuLi}$ & $1.0 \times 10^{-3}$ & 21900 & 1.45 & 91 \\
\hline
\end{tabular}

Table 2. Results of the anionic homo-polymerization of 4-DMVSBCB

\subsubsection{Poly (1-vinylsilylbenzocyclobutene)}

In comparison with 4-DMVSBCB, where the silicon atom bonds with benzene ring (Bz), 1DMVSBCB exhibit a structure change that the silicon atom bonds with cyclobutene $(\mathrm{Ct})$ (Scheme 5). This appeared slight structural change leads to a significantly alteration in polymerization behavior, especially for anionic polymerization. The anionic polymerization did occur for 1-DMVSBCB but required several days. In addition, it was noted that the polymerization did not proceed except using sec-BuLi with higher reactivity. The yield (60 70 wt.\%) and molecular weight (around $10000 \mathrm{~g} / \mathrm{mol}$ ) of poly (1-DMVSBCB) are lower than that of poly (4-DMVSBCB). These results indicated a lower polymerization activity of 1-DMVSBCB, which is possibly attributed to that the electron donating property of cyclobutene reducing the stability of carbon anion or radicals. DSC analysis showed that the initial cross-linking temperature of poly (1-DMVSBCB) is about $161{ }^{\circ} \mathrm{C}$, which is lower than that of poly (4-DMVSBCB), about $180{ }^{\circ} \mathrm{C}$. It once again supports that the substituting of cyclobutene would enable the tuning of ring-opening temperature of benzocyclotuene. Unfortunately, poly (1-DMVSBCB) shows lower initial decomposition temperature and thermal stability relative to poly (4-DMVSBCB). Apparently, 1-subsitution would also lead to a reduction in the stability of derived dibenzocyclooctadiene except for that of 
cyclobutene. Hence, it may be a big challenge to simultaneously achieve high thermostability of cross-linked polymer and low cross-linking temperature.

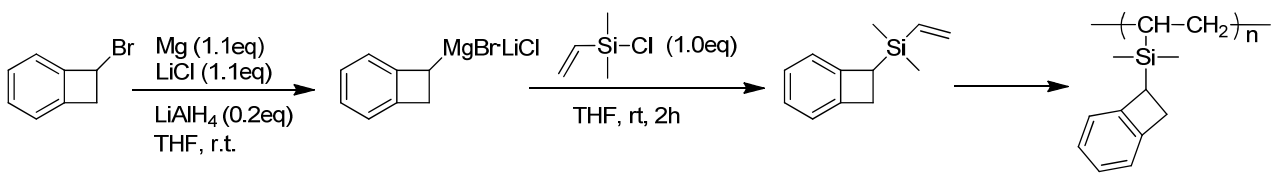

Scheme 5. Synthesis route of 1-DMVSBCB and poly (1-DMVSBCB)

\section{Other vinyl-BCB polymers}

Benzocyclobutene-4-yl acrylate exhibits effective homo-polymerization and copolymerization by free radical methods as its similar property with methyl acrylate (Scheme 6). BCB units are able to be incorporated into a series of polyarylates or other polyolefins by copolymerization. Related studies (19) showed that the incorporation ratio of 4-ABCB in copolymer could reach $6.7 \mathrm{~mol} \%$ when using $4.7 \mathrm{~mol} \%$ feed ratio of 4 -ABCB. This result indicates that $4-\mathrm{ABCB}$ exhibit higher polymerization activity than MA.

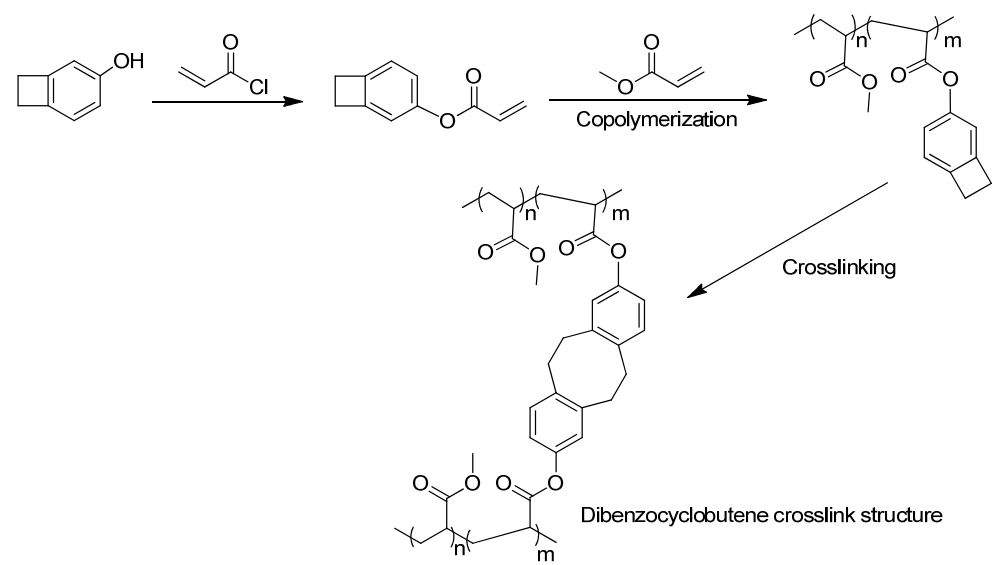

Scheme 6. Preparation and cross-linking of 4-ABCB/MA copolymer.

In recent years, apart from its conventional application in coating, adhesive, etc, polyarylates have been studied for use in a wide range of other areas such as gas separation membranes, hydrogels, tissue engineering, drug delivery, dentistry, and shape-memory materials for cardiovascular applications. Particularly, the potential applications of polyarylates as shape memory materials, which are capable of memorizing temporary shapes and recovering their permanent shape upon thermal activation, have attained much attention (21). For polyarylates to possess shape memory properties, it is necessary to have a permanent network which can be achieved via chemical or physical cross-linking. Interestingly, the cross-linking of polyarylates by the reaction of $\mathrm{BCB}$ generates nanoparticles which serve as physical cross-linkers (19). As a result, physical cross-links were generated, apart from chemical cross-links. This unique cross-linking architecture 
would be beneficial to broaden the list of cross-linking methods used in the production of SMPs.

Benzocyclobutene-1-yl acrylate (1-ABCB) exhibits similar radical polymerization behavior with $4-\mathrm{ABCB}$ (Scheme 7). 1-ABCB also shows higher activity than MA as 1-ABCB/MA copolymerization results indicate that $1-\mathrm{ABCB} / \mathrm{MA}$ ratio in copolymer is higher than 1$\mathrm{ABCB} / \mathrm{MA}$ feed ratio. Interestingly, the initial ring-opening temperature of poly $(1-\mathrm{ABCB})$ is approximately $170{ }^{\circ} \mathrm{C}$, which is lower than that of 4-subsituted BCB.

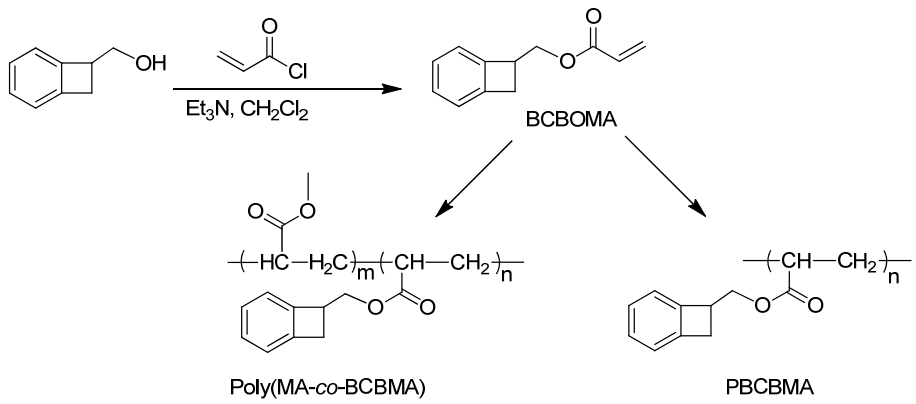

Scheme 7. Synthesis route of 1-ABCB and homo/copolymers of 1-DMVSBCB.

\section{Benzocyclobutene-siloxane resins}

\section{Benzocyclobutene-linear siloxane}

The purpose of designing benzocyclobutene-siloxane structure is to introduce the excellent thermostability of siloxane materials into BCB structure. The benzocyclobutene-siloxane materials are expected to possess integrated properties of polysiloxane and BCB related materials. As described above, one representative resin developed earlier is poly (DVS$\mathrm{BCB}$ ). However, poly (DVS-BCB) shows poor controllability of the property of precrosslinked polymer used for film-forming. To solve this problem, Yang et al. have prepared a mixture of poly (4-VBCB-co-St) and poly (DVS-BCB) (22). Cross-linking of this blend generates a complicated network (Scheme 8). By incorporating poly (4-VBCB-co-St), the viscosity was improved and slight cross-linking would afford polymers with adequate viscosity for film-forming. In this work, two poly (4-VBCB-co-St)s with 4-VBCB/St ratios of 1:3 and 1:5 were used. Correspondingly, the $5 \%$ weight loss temperature of the resulted cross-linked polymers is around $410^{\circ} \mathrm{C}$ and $400{ }^{\circ} \mathrm{C}$, respectively.

On the other hand, the application of poly (DVS-BCB) have been limited largely in several standard interconnects due to their lower thermal stability and higher fragility relative to polysiloxanes. It implies that a simple combination of siloxane and BCB structure scarcely affords materials with high performance as expected. Structural analysis shows that the relatively low thermostability of poly (DVS-BCB) may be due to the incomplete crosslinking of DVS-BCB, the presence of residue ethylene moieties which are prone to take oxidation reaction, or the $\mathrm{BCB}$-siloxane alternating copolymerization structure which destroys the thermally stable Si-O-Si backbone. Yang et al. have prepared a new siloxane-BCB monomer, 

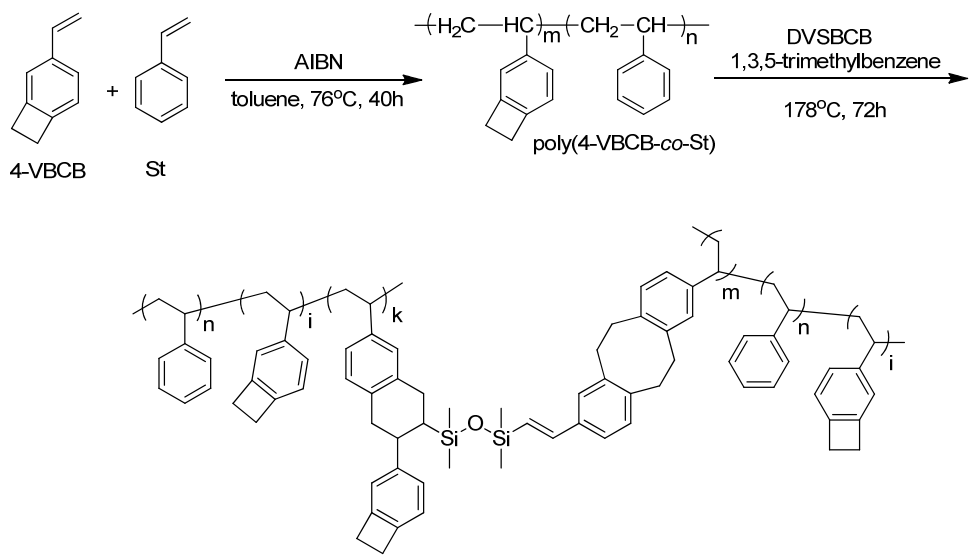

Scheme 8. Preparation of poly (4-VBCB-co-St) and the conetwork structure with DVSBCB.

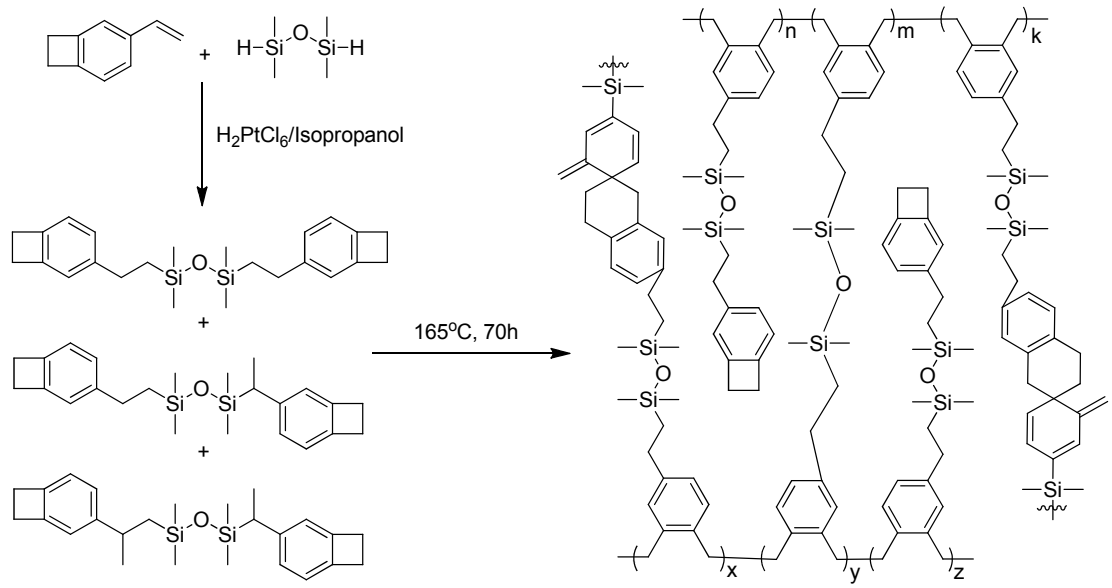

Scheme 9. Synthesis route to DES-BCB and the cross-linked poly (DES-BCB)

1,1,3,3-tetramethyl-1,3-bis[2'-(4'-benzocyclobutenyl)]vinyldisiloxane (DES-BCB) (Scheme 9). Ethyl was introduced instead of vinyl in DVS-BCB, which might enhance the thermal oxidation stability and decrease the fragility of cross-linked polymers simultaneously. However, TGA of poly (DES-BCB) shows a relatively inferior thermal stability with $5 \%$ weight loss temperature of $380^{\circ} \mathrm{C}$, indicating that alkene oxidation would not be responsible for the inferior thermal stability of poly (DVS-BCB).

In their further work, a new BCB-siloxane polymer with polysiloxane backbone and $\mathrm{BCB}$ pendant groups was reported. This polymer was prepared by $\mathrm{Pt} / \mathrm{C}$ or $\mathrm{H}_{2} \mathrm{PtCl}_{6}$ catalyzed hydrosilylation between poly (methylhydrosiloxane) and 4-DMVSBCB or poly (methylvinylsiloxane) and 4-dimethylhydrosilylbenzocyclobutene (4-DMHSBCB) (Scheme 10). The incorporation ratio of 4 -DMVSBCB pendant groups is tunable, and the maximum ratio is up to $72 \%$. The corresponding cross-linked BCB-siloxane resins exhibit an initial 
decomposition temperature of $429^{\circ} \mathrm{C}$, showing a superior thermal resistance over poly (DVS-BCB) resins and other common polysiloxanes.

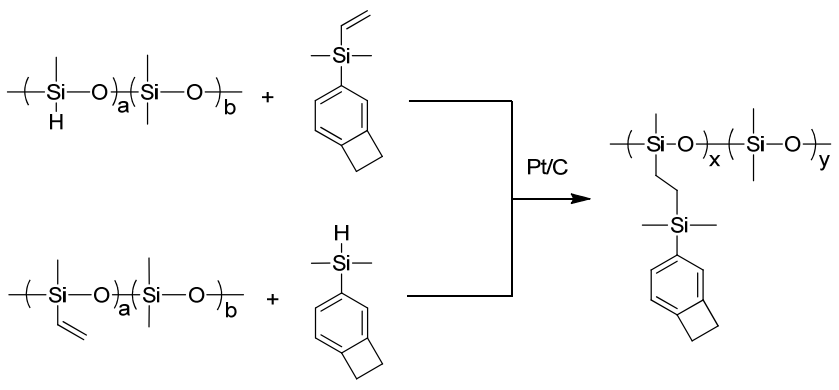

Scheme 10. Preparation of polysiloxane with BCB containing pendant groups.

Fan et al (23) have prepared two novel siphenylene-siloxane copolymers bearing latent reactive $\mathrm{BCB}$ groups by polycondensation procedure of 1,4-bis (hydroxydimethylsilyl)benzene with bis (dimethylamino) methyl $\left(4^{\prime}-\right.$ benzocyclobutene)silane and bis(dimethylamino)methyl[2'-(4'benzocyclobutenyl)vinyl]silane (Scheme 11). The BCB-based monomers were synthesized from Grignard and Heck reaction, respectively. Copolymers with high molecular weight $(M w)$ of 1.96 and $1.61 \times 10^{5} \mathrm{~g} / \mathrm{mol}$ have been obtained which were readily converted to crosslinked films upon heating. The copolymer originated from SiViBu showed a big exotherm peak starting at about $230^{\circ} \mathrm{C}$. This temperature is similar with DVS-BCB but is higher than that of copolymer originated from $\mathrm{SiBu}$. It may be attributed to their different cure mechanism. The cross-linking of $\mathrm{SiBu}$ proceeds via a Diels-Alder reaction of two oquinodimethanes, while that of $\mathrm{SiViBu}$ and $\mathrm{SiBu}$ proceeds via a Diels-Alder reaction of $o$ quinodimethane as a diene and vinyl group as a dienophile. The final resins exhibited excellent thermal stability with high temperatures at $5 \%$ weight loss $\left(553\right.$ and $526{ }^{\circ} \mathrm{C}$ in $\mathrm{N}_{2}$, 530 and $508{ }^{\circ} \mathrm{C}$ in air) owing to the incorporation of the benzene unit into the mainchains of the copolymers. The films of cured copolymers also demonstrated low dielectric constants of 2.66 and 2.64 and low dissipation factors of 2.23 and $2.12 \times 10^{-3}$.

In their further work (24), two new methylphenylsiloxane monomers terminated with crosslinkable benzocyclobutene functionalities, 1,1,5,5-dimethyldiphenyl-1,1,5,5-di[2'-(4'benzocyclobutenyl)vinyl]-3,3-diphenyltrisiloxane (BCB-1) and 1,1,3,3-dimethyl-diphenyl1,1,3,3-di[20-(40-benzocyclobutenyl)vinyl]disiloxane (BCB-2), were prepared (Scheme 12). These two monomers possess similar structure with DVS-BCB. Correspondingly, they show a simultaneous polymerizing and cross-linking process. Cured BCB-1 and BCB-2 exhibited good thermal stability $\left(T_{5} \%>472{ }^{\circ} \mathrm{C}\right.$ both under $\mathrm{N}_{2}$ and air), which is better than that of DVS$\mathrm{BCB}$ resins and poly (4-DMVSBCB). From the comparison among DVS-BCB, BCB-1 and $\mathrm{BCB}$-polysiloxane resins, it can be seen that elongating the length of polysiloxane chains and incorporating rigid functional groups on $\mathrm{Si}$ are beneficial for enhancing thermal stability. BCB-1 and BCB-2 resins also demonstrated low dielectric constants (2.69 and 2.66) and low dissipation factors of 2.23 and $2.12 \times 10^{-3}$. 

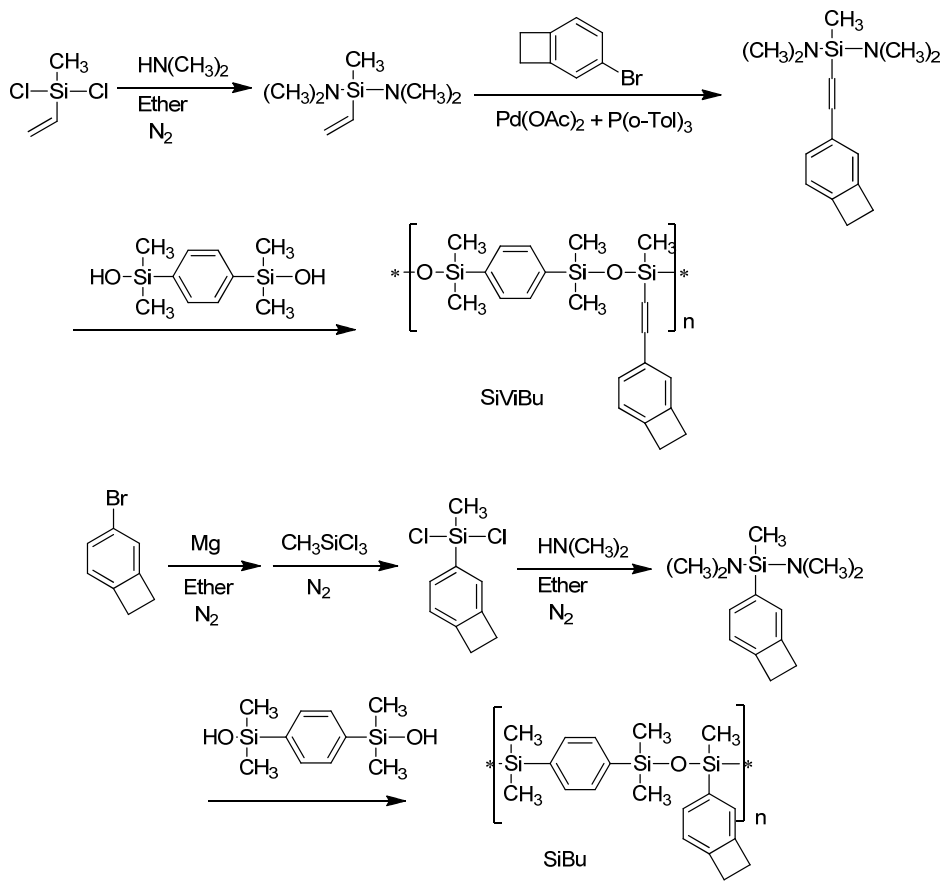

Scheme 11. Synthesis route to copolymers originated from $\mathrm{SiViBu}$ and $\mathrm{SiBu}$, respectively.

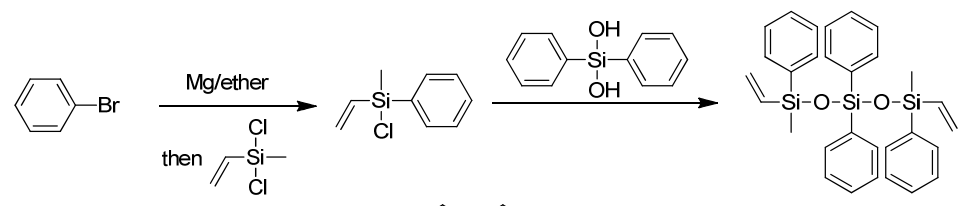

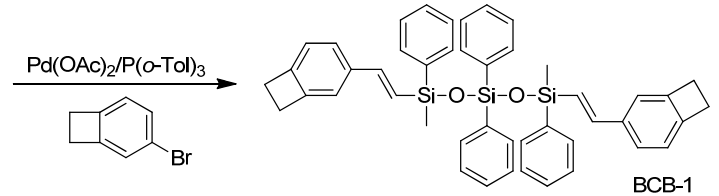

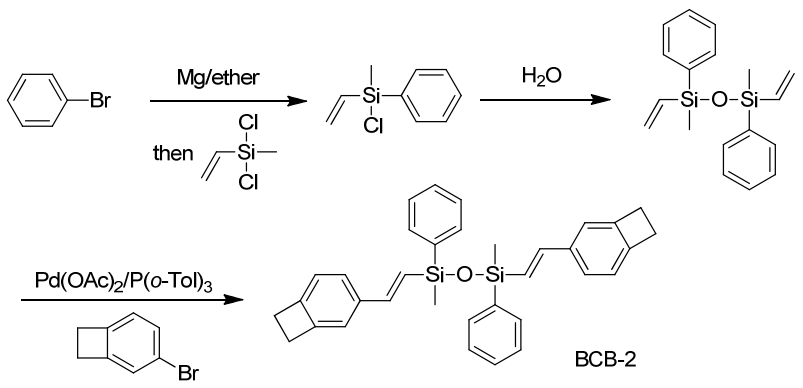

Scheme 12. Synthesis route to the monomers BCB-1 and BCB-2. 


\section{The application of BCB-based materials}

\subsection{Low dielectric materials}

The performance of integrated circuit devices, historically limited by characteristics of the transistors, has become limited by the back-of-end-of-line (BEOL) signal delay of interconnect caused by wire capacitance, crosstalk and power dissipation. As one of the major technological strategy to decrease signal delay, developing new generation of low dielectric materials as alternatives of $\mathrm{SiO}_{2}$ have received much attention in recent years. Besides low dielectric constant, a qualified dielectric materials must satisfy other requirements, including high thermal stability, high mechanical strength, high breakdown field above $2 \mathrm{MV} \mathrm{cm}^{-1}$, low moisture absorption, low thermal swelling coefficient or film stress, good adhesion to various matrix, ease of damascence processing or etching, reactive inertness, good film forming property, etc. Among kinds of the accessible alternatives, some polymers with outstanding and promising mechanical and physicochemical properties have been widely studied (25). In the past few years, the studies were mainly concentrated on the following polymers: polyimides, $\mathrm{BCB}$ resins, poly (binaphthylene ether), polydiphenyl, polyquinolines, polynorbornene, silicon-containing polymers and fluorinated polymers.

Although several potential applications were discovered for BCB-based materials, only the use as low dielectric materials was accessible for application until now. One of the reasons for the intense research on BCB-based low dielectric materials is that the thermal activation would generate a highly reactive intermediate to form clean products, which are beneficial for designation of high precision curing processes. The application of $\mathrm{BCB}$ resins in microelectronic should start from Cyclotene (26). So far, several formulations of these BCBbased resins have been commercialized for thin-film dielectric applications and are currently used in the fabrication of liquid crystal displays, GaAs integrated circuit interconnect, interlayer connection and chip bumps. Although BCB-based resins displayed several advantages over many other polymeric materials, their use in standard interconnect is limited by their relatively low thermal stability than the required process temperatures.

Table 2 lists the thermal and dielectric performances of BCB-based materials reported in the past few years. Most of them show low dielectric property with dielectric constant below 2.65 , it is worth noting that poly (4-DMVSBCB) possesses the lowest dielectric constant probably due to the absence of polar atoms in this carbosilane structure. Moreover, these new BCB-based materials show higher thermal stability except poly (4-vinylBCB). Most of them could be considered as potential alternatives of poly (DVS-BCB).

\subsection{Vinyl-BCB used for the functionalization of Carbon Nanotube}

The methods that have been explored for the functionalization of the $\mathrm{sp}^{2}$ network of the carbon nanotubes (CNTs) include addition reactions of azomethine ylides, bromomalonates, nitrenes, nucleophilic carbenes, and free radicals. In 2007, Baskaran et al. prepared functionalized CNTs by [4+2] cycloaddition of BCB for the first time (27). The stained ring of 


\begin{tabular}{lccc}
\hline & $\begin{array}{c}\mathrm{T}_{0} \\
\left({ }^{\circ} \mathrm{C}\right)\end{array}$ & $\begin{array}{c}\mathrm{T}_{5} \% \\
\left({ }^{\circ} \mathrm{C}\right)\end{array}$ & $\begin{array}{c}\text { Dielectric } \\
\text { constant }\end{array}$ \\
\hline Poly (DVSBCB) & 370 & & 2.65 \\
Poly (4-vinylBCB) & $\sim 350$ & & 2.58 \\
Poly (4-DMVSBCB) & 380 & 428 & $<2.45$ \\
Poly (DES-BCB) & & 380 & \\
Trisiloxane-BCB & & $>472$ & $2.66 \sim 2.69$ \\
poly (SiViBu) & & 553 & 2.66 \\
poly (SiBu) & & 526 & 2.64 \\
Polysiloxane-BCB & 427 & 493 & \\
\hline
\end{tabular}

Table 3. Thermal and dielectric performance of several BCB-based materials.

the $\mathrm{BCB}$ is susceptible to thermal ring opening to form o-quinodimethane, which can undergo [4+2] cycloaddition exclusively with 6-6 bonds of fused aromatic rings. In an attempt to add reactive organic groups on the surface of CNTs, several substituted BCBs were reacted with CNTs (28-29). Vinyl substituted BCBs, typically1-phenyl-1benzocyclobutene ethylene (BCB-DPE), are of particular useful because they can be attached on the surface of CNTs serving as anionic initiators for olefins polymerization. In a further work of Baskaran et al (28), the surface of multi walls carbon nanotubes (MWNTs) was modified via covalent attachment of BCB-polyethylene (BCB-PE) through Diels-Alder reaction at $235{ }^{\circ} \mathrm{C}$ (Scheme 13). TGA shows that MWNTs- $g$-(BCB-PE)n has $54 \mathrm{wt} \%$ precursor initiator. Anionic surface-initiated polymerization of styrene has been performed using such MWNTs functionalized with anionic initiators. Polymer grafted nanocomposites, MWNTs$g-(B C B-P S) n$, contains a very high percentage of hairy polymer with a small fraction of MWNTs (<1 wt \%). Size exclusion chromatography revealed a broad molecular weight distributions (1.3 1.8) of the polymer grafted MWNTs.
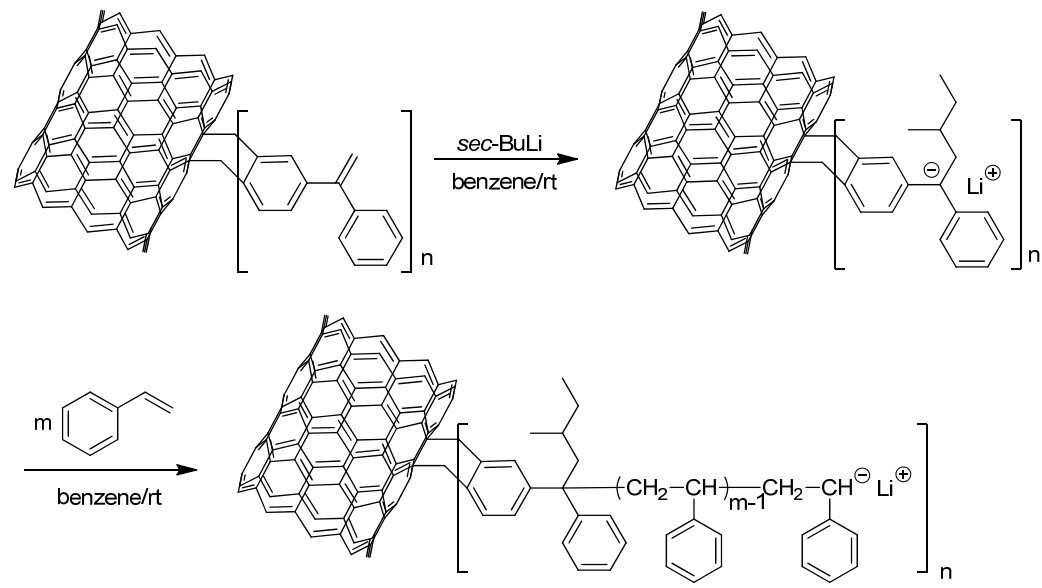

Scheme 13. Surface functionalization of CNT by 1-phenyl-1-benzocyclobutene ethylene which initiates anionic polymerization of styrene. 


\subsection{Applications in Organic Light Emitting Diodes (OLED)}

OLEDs have attained much research interest in the past few decades due to their promising applications in full color displays and solid-state lighting. Device fabrication, involving the construction of hole-transporting layer (HTL), phosphorescent emitting layer (EL), and electron-transporting layer (ETL) multilayers structure, shows great impact on the ultimate performance. There are usually two methods to fabricate the layers. One is high vacuum vapor deposition of small molecules, and another is solution processing of polymers or dendrimers. The solution processing technique presents advantages in thermostability. However, it requires that each deposited layer must resistant to the solvent that used to deposit subsequent layers. Perhaps the most elegant strategy to address these issues involves the development of cross-linkable materials. To date, most of the reported thermally cross-linkable hole-transport materials are based on perfluorocyclobutane (PFCB) cross-linking unit. Recently, a novel thermally cross-linkable hole-transporting polymer for solution processible multilayer OLEDs was designed (30). This amorphous polymer was prepared from the radical copolymerization of vinyl-BCB with 4-[N-(4-Vinylphenyl)-N-(4methylphenyl) amino]-4'-[N-phenyl-N-(4-methylphenyl) amino]biphenyl initiated by AIBN (Scheme 14). It contains $\sim 9 \mathrm{~mol} \%$ of $\mathrm{BCB}$ with the $T_{g}$ of near $175{ }^{\circ} \mathrm{C}$. Cross-linking of the copolymer at $200{ }^{\circ} \mathrm{C}$ led to insoluble polymer films with a smooth surface. Solution processed multilayer light emitting diodes were prepared using this new BCB-inducing cross-linked polymer as a hole-transporting layer. These light emitting diodes exhibited high performance with $10.4 \%$ external quantum efficiency at a brightness of $350 \mathrm{~cd} / \mathrm{m}^{2}$.

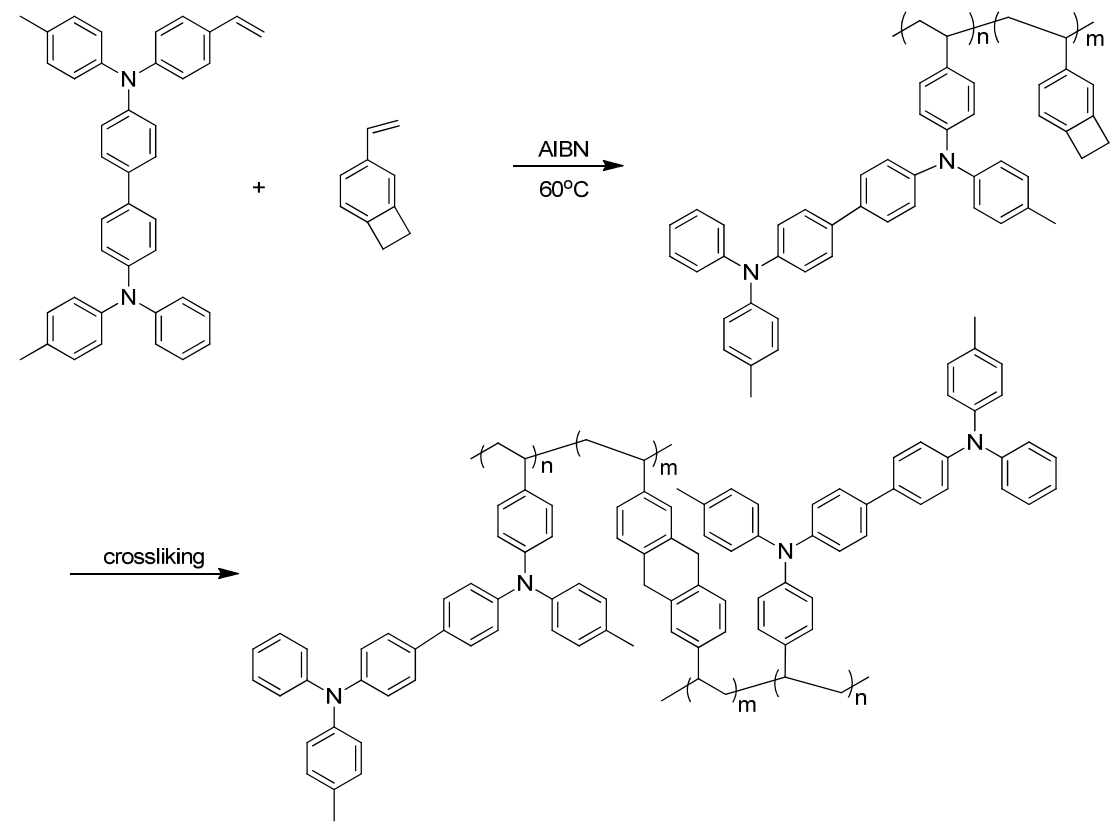

Scheme 14. Preparation and cross-linking of TPD-BCB copolymers. 


\subsection{Applications in organic field-effect transistors}

Organic field-effect transistors (FETs) are promising in enabling disposable electronics and flexible electronics technologies. Fabricating ultrathin defect-free gate dielectric layer with a high quality interface to the semiconductor layer is crucial for polymeric FETs. In recent years, several polymer dielectric materials including spin-on silesquioxanes, photoresist, polyimide and poly (vinylphenol) (PVP) were investigated. However, these polymers are not wholly satisfactory because they still cannot give conformal and pinhole-free films in the sub-100-nm regime. Consequently, present spin-on polymer dielectrics are typically limited to more than $300 \mathrm{~nm}$ in thickness, thus demanding a large gate voltage to operate. Recent works show that DVS-BCB possesses most of the requisite gate dielectric properties (31). Furthermore, defect-free films down to a few tens of nanomaters in thickness are attainable by a simple solution casting technique. The fabricated devices can operate at a low voltage with a field-effect mobility of few $10^{-4} \mathrm{~cm}^{2} / \mathrm{Vs}$. They can be continuously operated at $120^{\circ} \mathrm{C}$, showing a good operating stability.

\section{Perspective of $B C B$ related chemistry and materials}

\subsection{Potential polymerization methods}

Although most of the vinyl-BCBs can be polymerized by anionic and radical polymerization methods, their copolymerization with styrene, MMA, ethylene and so on encountered a big challenge as the great difference polymerization activity between them. Coordination polymerization method as a powerful tool has promoted a great development of polymer chemistry in the last decades. However, the coordination polymerization of vinyl-BCB monomers has not been reported yet. In fact, most of these monomers mentioned above are capable of polymerizing through coordination polymerization in terms of its similar structure with styrene and acrylate. Of course, the coordination polymerization of vinylsilylBCB, such as 4-DMVSBCB and 1-DMVSBCB, is more difficult due to the Lewis acid property of silicon element and the large steric hindrance. The coordination polymerization of several simple vinylsilane compounds, indeed, has been studied. The firstly used catalysts were Ziegler-Natta catalysts, which however failed to induce accepted polymerization performance. An acceptable result was obtained till 2008 when Nomura et al has demonstrated a successful copolymerization of vinyltrialkylsilanes with ethylene catalyzed by nonbridged half-titanocenes (32). Thus, in this regard, a large number of works including new high-efficiency catalysts developing and in-depth polymerization behavior and mechanism studying are highly desired in the field of vinyl-BCB related polymer chemistry.

Earlier work demonstrated that free radical method could not initiate the polymerization of vinyl-silylBCB. Nevertheless, it does not mean that the radical polymerization definitely fails to give products with high molecular weight and poor yields. Theoretically, the living free radical method could stabilize the initiated monomer free radicals, thus extending the lifetime of them and consequently enhancing polymerization activity. In addition, living 
radical polymerization of silyl-functionalized vinyl monomers with large steric hindrance, for example vinylcyclicsilazane, has been indeed reported (33). The use of RAFT agents with anionic characteristic displayed excellent activity, giving the product with molecular weight up to $11300 \mathrm{~g} / \mathrm{mol}$. Thus, further studies regarding the radical polymerization of vinylsilylBCB should include the investigation of living polymerization, such as RAFT and ATRP. Moreover, these methods can also be used for the polymerization of vinyl-BCB to afford improved and controlled molecular weight, enabling the generation of random even block copolymers.

\subsection{Low temperature cross-linking}

Although the cross-linking by BCB ring-opening reaction presents several superiorities over common cross-linking ways, its high ring-opening temperature would induce the deformation of films due to the generally low $T_{g}$ of the linear BCB precursors, thus limiting its use in some extent. Previous work demonstrated that the ring-opening temperature of cyclobutene is tunable by incorporating variety of substituents on cyclobutene (34). In particular, when utilizing the electron-donating substituents, the stability of cyclobutene and the ring-opening temperature was pronounced decreased. Recent work (35) even reveals that when placed within long polymer strands, the trans and cis isomers of a 1, 2-disubstitued benzocyclobutene undergo an ultrasound-induced electrocyclic ring opening at 6-9 ${ }^{\circ} \mathrm{C}$ (Scheme 15). Overall, developing new BCB structures, which enable lower opening temperature, is great important for improving the performance and expanding the application range of the vinyl containing $\mathrm{BCB}$ polymeric resins and also other $\mathrm{BCB}$ resins. Moreover, the cross-linking structure ascribed from the ring-opening reaction of 1-substitued or 1, 2-disubstituted benzocyclobutene is still unclear and needs to be further studied.
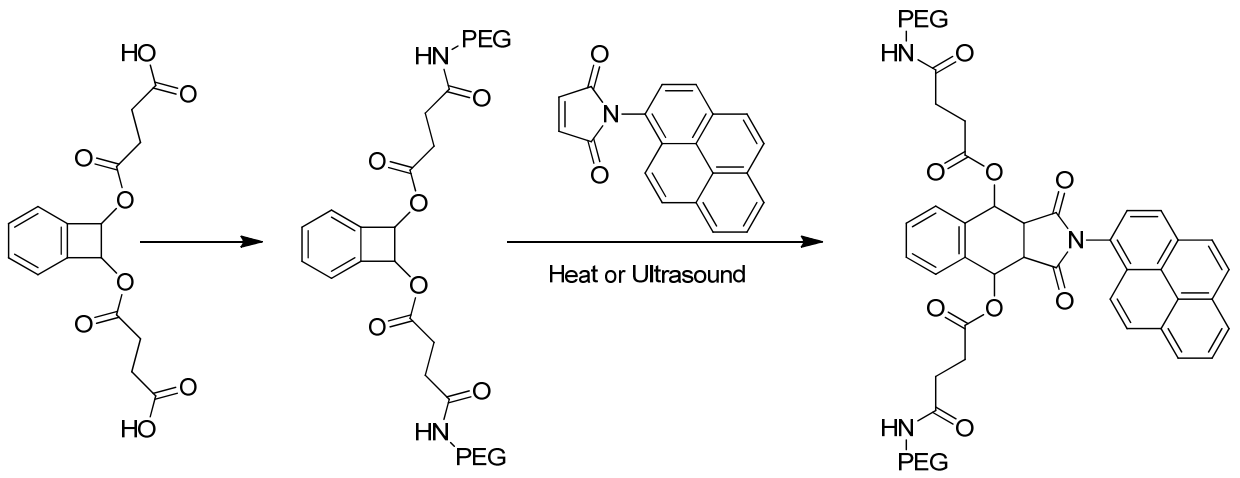

Scheme 15. Preparation and reaction of mechanosensitive polymers.

\subsection{Nanomaterials}

Currently, the nanomaterials with unique chemical structure and corresponding high performance and attractive functions have been attracted increased attention. Hence, 
exploiting vinyl-BCB based materials with nanostructure would be an interesting research topic because of the cross-linkable characteristic upon heating and the unique cross-linking behavior of BCB. Based on the well-established in-situ methods to construct nanostructures from polymerization of olefins, similar nanostructures based on poly (vinyl-BCBs) could be easily constructed. These BCB-based nanomaterials can be potentially used as adsorption materials, optic-electronic materials, coating materials, and so on.

More recently, Harth et al. present a new BCB based cross-linking structure that allows a 100 ${ }^{\circ} \mathrm{C}$ lower ring-opening temperature in comparison with conventional ones (36). They found that the incorporation of BCB based cross-linking unit by copolymerization shows a limited molecular weight control. Thus, as an alternative, they explored a grafting-on method by grafting a low-temperature cross-linking unit, 2-(1,2-dihydrocyclobutabenzen-1yloxy)ethanol, onto a linear acrylate polymer backbone (Scheme 16). To afford the required end group functionality, a convenient method was adopted to convert the alcohol to an amine for grafting onto the polymer. The polyacrylate was prepared via reversible additionfragmentation transfer (RAFT) polymerization. A polymeric precursor with a $75,000 \mathrm{Mw}$ polyacrylate with a PDI of 1.21 was obtained using AIBN catalyst towards the RAFT initiator. The amine was grafted to poly (acrylic acid) through chloroformate activation chemistry and the $5 \%$ conversion ratio of the carboxylic acid functional groups to functionalized benzocyclobutene units was obtained. Monitoring by ${ }^{1} \mathrm{H}$ NMR show that the ring-opening was initiated at the temperature higher than $100{ }^{\circ} \mathrm{C}$ and the optimal
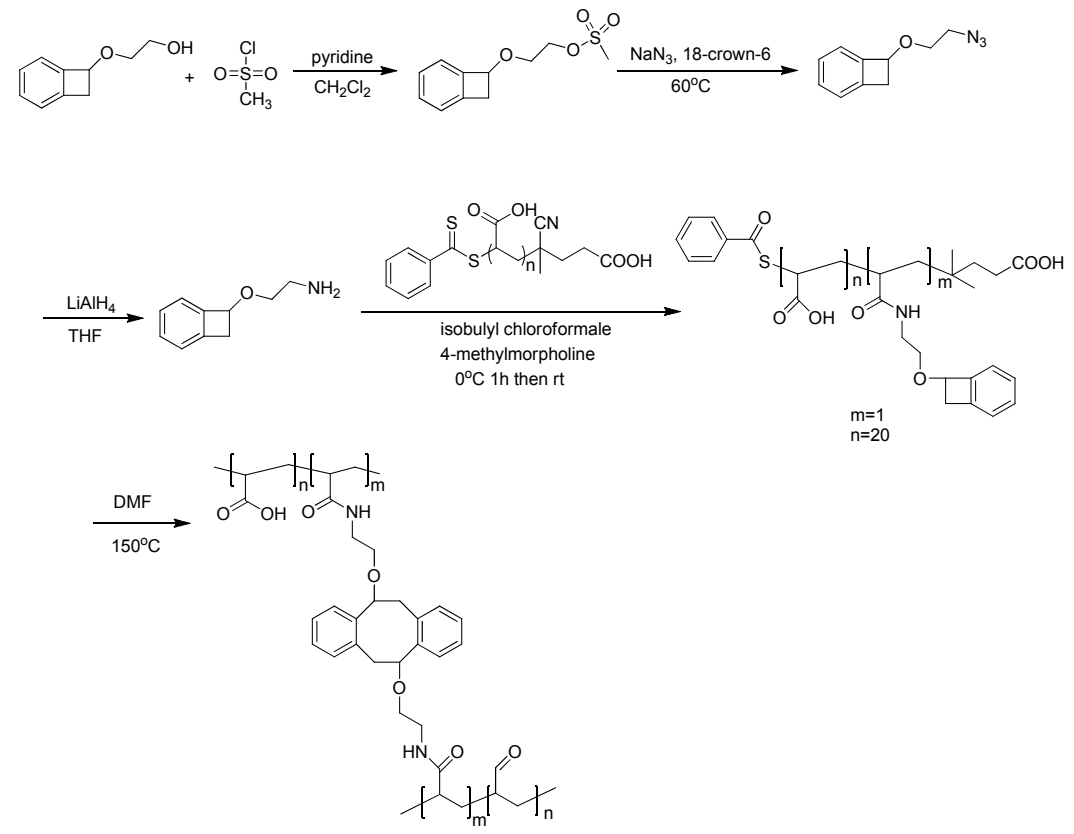

Scheme 16. Synthesis of the amine functionalized low-temperature benzocyclobutene and Grafting of this BCB amine onto poly (acrylic acid) 
temperature is above $130{ }^{\circ} \mathrm{C}$ at which the polymeric precursor could be fully converted. Dynamic Light Scattering (DLS) and TEM confirmed the formation of nanoparticles from the cross-linking of linear copolymers. TEM and DLS suggest that the mean diameter of nanoparticles is approximately $4.3 \pm 0.8$ and $7.3 \pm 0.5 \mathrm{~nm}$, respectively.

\section{Conclusions}

In the past few years, the approach to the utilization of benzocyclobutenes to construct polymers has experienced significant change. BCB-related polymers based on two-step cross-linking technology, represented by poly (vinyl-BCB)s, has brought remarkable improvement in controllability of materials structures and properties. Apart from this, their excellent polymerization compatibility with traditional olefins allows the convenient incorporation of BCB functionalities, significantly improving properties of original polymers and extending the potential application of BCB-related polymers. Besides, a series of BCBsiloxane polymers with novel structures exhibiting excellent thermal stability and low dielectric properties have been prepared. Indeed, some of these polymers are promising alternatives of poly (DVSBCB) and would be widely used in microelectronic devices. These exciting works are just a beginning. A new window has been opened for $\mathrm{BCB}$ related science and technology. The future development will mainly focus on the following areas: 1) searching for more suitable BCB-related dielectric materials; 2) utilizing $\mathrm{BCB}$ to construct novel structures and improve performance of original materials; 3) exploiting more applications suitable for BCB-related polymers.

\section{Author details}

Junxiao Yang, Yawen Huang and Ke Cao

School of Material Science and Engineering, Southwest University of Science and Technology, P. R. China

\section{Acknowledgement}

The authors would like to thank the financial support through the National Natural Science Foundation of China-NSAF and China-NSFC, and Open Project of State Key Laboratory Cultivation Base for Nonmetal Composites and Functional Materials for Southwest University of Science and Technology. Prof. Yang would like to thank students from his research for their contributions.

\section{References}

[1] Kirchhoff, R.A.; Bruza, K.J. (1993) Benzocyclobutenes in polymer synthesis. Progress in Polymer Science, 18, 85-185.

[2] Farona, M.F. (1996) Benzocyclobutenes in Polymer Chemistry. Progress in Polymer Science, 21, 505-555. 
[3] Sakellariou, G.; Baskaran D.; Hadjichristidis, N.; Mays, J.W. (2006) Well-defined poly (4vinylbenzocyclobutene): synthesis by living anionic polymerization and characterization. Macromolecules, 39, 3525-3530.

[4] Mehta, G.; Kotha, S. (2001) Recent chemistry of benzocyclobutenes. Tetrahedron. 57, 625659.

[5] Sadana, A.K.; Saini, R.K.; Billups, W.E. (2003) Cyclobutenes and related compounds. Chemistry of Review. 103, 1539-1602.

[6] Boaen, N.K.; Hillmyer, M.A. (2005) Post-polymerization functionalization of polyolefins. Chemical Society Reviews. 34, 267-275.

[7] Leibfarth, F.A.; Schneider, Y.; Lynd, N.A.; Schultz, A.; Moon, B.; Kramer, E.J.; Bazan, G.C.; Hawker, C.J. (2010) Ketene functionalized polyethylene: control of cross-link density material properties. Journal of the American Chemical Society. 132, 14706-14709.

[8] Endo. T. Koizumi T. Takata T. Chino K. (1995) Synthesis of poly(4vinylbenzocyclobutene) and its reaction with dienophiles. Journal of polymer science, Part A. 33, 707-715.

[9] Harth, E.; Horn, B. V.; Lee, V. Y.; Germack, D. S.; Gonzales, C. P.; Miller, R. D.; Hawker, C. J. (2002) A facile approach to architecturally defined nanoparticles. Journal of the American Chemical Society. 124, 8653-8660.

[10] So, Y.H.; Hahn, S.F.; Li, Y.; Reinhard, M.T. J. (2008) Styrene 4-vinylbenzocyclobutene copolymer for microelectronic application. Journal of polymer science, Part A. 33, 707-715.

[11] Leiston-Belanger, J.M.; Russell, T.P.; Drockenmuller, E.; Hawker, C.J. (2005) A thermal and manufacturable approach to stabilized diblock copolymer templates. Macromolecules, 38, 7676-7683.

[12] Chino, K.; Takata, T.; Endo, T. (1998) Polymerization of o-Quinodimethanes. III. Polymerization of o-Quinodimethanes Bearing Electron-Withdrawing Groups Formed in Situ by Thermal Ring-Opening Isomerization of Corresponding Benzocyclobutenes. Journal of polymer science, Part A. 37, 1555-1563.

[13] Hahn, S.F.; Martin, S.J.; McKelvy, M.L. (1992) Thermally Induced Polymerization of an Arylvinylbenzocyclobutene Monomer. Macromolecules. 25, 1539-1545.

[14] Pyun, J.; Tang, C.B.; Kowalewski, T.; Frechet, J.M.J.; Hawker, C.J. (2005) Synthesis and Direct Visualization of Block Copolymers Composed of Different Macromolecular Architectures. Macromolecules, 38, 2674-2685.

[15] Kim, Y.; Pyun, J.; Frechet, J.M.J.; Hawker, C.J.; Frank, C.W. (2005) The Dramatic Effect of Architecture on the Self-Assembly of Block Copolymers at Interfaces. Langmuir. 21, 10444-10458.

[16] Ryu, D.Y.; Shin, K.; Drockenmuller, E.; Hawker, C.J.; Russell, T.P. (2005) A generalized approach to the modification of solid surfaces, Macromolecules. 308, 236-239.

[17] Ryu, D.Y.; Wang, J.Y.; Lavery, K.A.; Drockenmuller, E.; Satija, S.K.; Hawker, C.J.; Russell, T.P. (2007) Surface Modification with Cross-Linked Random Copolymers: Minimum Effective Thickness. Macromolecules. 40, 4296-4300.

[18] Yang, J.X.; Liu, S.C.; Zhu, F.H.; Huang, Y.W.; Li, B.; Zhang, L. (2011) New Polymers Derived from 4-Vinylsilylbenzocyclobutene Monomer with Good Thermal Stability, 
Excellent Film-Forming Property, and Low-Dielectric Constant, Journal of Polymer Science, Part A, 49, 381-391.

[19] Yang, J.X.; Xie, L.Q.; Zhu, F.H.; Sui, H.B.; Li, H.L.; Huang, Y.W. (2011) Incorporation of Benzocyclobutene Cross-Linkable Moieties in Poly(Methyl Acrylate): A Novel Approach to Shape-Memory Polymers Accompanied with Microphase Separation, Journal of Macromolecular Science, Part B. 50, 2129-2139.

[20] Xie, L.Q.; Yang, J.X.; Zhu, F.H.; Yang, H.J.; Liu, C.L.; Zhang, L. (2010) Preparation and Polymerization of Benzocyclobuten-4-yl Acrylate Monomer, Chinese Journal of Polymer Science, 28, 877-885.

[21] Smith, K.E.; Parks, S.S.; Hyjek, M.A.; Downey, S.E.; Gall, K. (2009) The effect of the glass transition temperature on the toughness of photopolymerizable (meth)acrylate networks under physiological conditions. Polymer. 50, 5112-5123.

[22] Xu, Y.W.; Zhu, F.H.; Xie, L.Q.; Yang, J.X.; Zhang, L.; Xie R.G. (2010) Synthesis and property studies of oligomer obtained from the reaction of 4-vinylbenzocyclobutene and styrene with divinyl tetramethyl disiloxane-bisbenzocyclobutene, e-Polymers, 013.

[23] Zuo, X.B.; Chen, J.S.; Zhao, X.J.; Yang, S.Y.; Fan, L. (2008) Synthesis and Characterization of Siloxane Resins Derived from Silphenylene-Siloxane Copolymers Bearing Benzocyclobutene Pendant Groups, Journal of Polymer Science, Part A, 46, 78687881.

[24] Zuo, X.B.; Yu, R.L.; Shi, S.; Feng, Z.H.; Li, Z.P.; Yang, S.Y.; Fan, L. (2009) Synthesis and Characterization of Photosensitive Benzocyclobutene-Functionalized Siloxane Thermosets, Journal of Polymer Science, Part A, 47, 6246-6258.

[25] Zhao X.Y. Liu H.J. (2010) Review of polymer materials with low dielectric constant. Polymer International, 59, 597-606.

[26] Martin S.J. Godschalx J.P. Mills M.E. Shaffer E.O. Townsend P.H. (2000) Development of a Low-Dielectric-Constant Polymer for the Fabrication of Integrated Circuit Interconnect. Advanced Material. 12, 1769-1778.

[27] Sakellariou, G.; Ji, H.N.; Mays, J.W.; Hadjichristidis, N.; Baskaran, D. (2007) Controlled Covalent Functionalization of Multiwalled Carbon Nanotubes using $\left[\begin{array}{ll}4 & +2\end{array}\right]$ Cycloaddition of Benzocyclobutenes. Chemistry of Materials. 19, 6370-6372.

[28] Sakellariou, G.; Ji, H.N.; Mays, J.W.; Baskaran, D. (2008) Enhanced Polymer Grafting from Multiwalled Carbon Nanotubes through Living Anionic Surface-Initiated Polymerization. Chemistry of Materials. 20, 6217-6230.

[29] Priftis, D.; Petzetakis, N.; Sakellariou, G.; Pitsikalis, M.; Baskaran, D.; Mays, J.W.; Hadjichristidis, N. (2009) Surface-Initiated Titanium-Mediated Coordination Polymerization from Catalyst-Functionalized Single and Multiwalled Carbon Nanotubes. Macromolecules. 42, 3340-3346.

[30] Ma, B.W.; Lauterwasser, F.; Deng, L.; Zonte, C.S.; Kim, B.J.; Frechet, J.M.J. (2007) New thermally cross-linkable polymer and its application as a hole-transporting layer for solution processed multilayer organic light emitting diodes. Chemistry of Materials. 19, 4827-4832. 
[31] Chua, L.L.; Ho, P.K.H.; Sirringhaus, H.N.; Friend, R.H. (2004) High-stability ultrathin spin-on benzocyclobutene gate dielectric for polymer field-effect transistors. Applied Physic Letters, 84, 3400-3402.

[32] Nomura, K.; Kakinuki, K.; Fujiki, M.; Itagaki, K. (2008) Direct precise functional group introduction into polyolefins: efficient incorporation of vinyltrialkylsilanes in ethylene copolymerizations by nonbridged half-titanocenes. Macromolecules. 41, 8974-8976.

[33] Nghiem, Q.D.; Nguyen, C.T.; Kim, D.P. (2008) Controlled/Living Radical Polymerization of Vinylcyclicsilazane by RAFT Process and Their Block Copolymers. Journal of Polymer Science. Part A. 46, 4594-4601.

[34] Matsuya, Y.; Ohsawa, N.; Nemoto, H. (2006) Accelerated Electrocyclic Ring-Opening of Benzocyclobutenes under the Influence of a $\alpha$-Silicon Atom. Journal of the American Chemical Society, 128, 412-413.

[35] Hickenboth, C.R.; Moore, J.S.; White, S.R.; Sottos, N.R.; Baudry, J.; Wilson, S.R. (2007) Biasing reaction pathways with mechanical force. Nature, 446, 423-427.

[36] Dobish, J.N.; Hamilton, S.K.; Harth, E. (2012) Synthesis of low-temperature benzocyclobutene cross-linker and utilization. Polymer Chemistry. 3, 857-860. 